\title{
CO Observations as a diagnostic of possible environmental effects ${ }^{1}$
}

\author{
Marcio A. G. Maia \\ Observatório Nacional - MCT, Rio de Janeiro, Brazil. \\ Duilia de Mello and Tommy Wiklind \\ Onsala Space Observatory, Onsala, Sweden.
}

\begin{abstract}
We have compared two samples of galaxies in very high and low density environments in order to study their physical properties and stablish any possible relation among them. This paper focus on the CO content as a diagnostic tool to examine galaxy properties such as the amount of molecular gas and the star formation efficiency. The similarities in star formation efficiency of the dense environments and the field galaxies suggest that the physical processes controling the formation of stars from the molecular gas are local rather than global.
\end{abstract}

\section{Introduction}

There is no doubt that the environment where galaxies reside plays a considerable role in galaxy evolution. Nevertheless, there are a few key questions which are still being debated. For instance, the environmental influences on the star formation properties of clusters of galaxies is far from clear-cut. The Coma spirals are similar to those in the Virgo cluster: deficient in atomic gas while the molecular gas properties are the same as for field spirals (eg., Casoli et al. 1991, 1996). In the Fornax cluster, Horellou et al. (1995) found no evidence for HI deficiency, but an unusual low fraction of molecular gas. In compact groups of galaxies - CG, entities with a space density of galaxies higher than in clusters, gravitational interactions should be even more important, but the results indicate differently. Even though CG present a high fraction of distorted galaxies (Mendes de Oliveira \& Hickson 1994), they do not show an enhancement in farinfrared (FIR) emission (Sulentic \& de Mello Rabaça 1993). The low HI content (Williams \& Rood 1987) and the normal CO content (Boselli et al. 1996, Leon et al. 1998) suggest that galaxies in CG might present less star-formation because they have less fuel. In pairs of galaxies, the scenario is different. The $\mathrm{CO}$ and far-infrared luminosities, are enhanced (Combes et al. 1994). However, whereas the star formation efficiency ( $\mathrm{SFE}=$ star formation rate per mass unit of molecular gas) is increased in strongly interacting/merging galaxies, the average SFE

\footnotetext{
${ }^{1}$ Based on observations at the $15 \mathrm{~m}$ Swedish ESO Submillimeter telescope, SEST, and at the $1.52 \mathrm{~m}$ ESO telescope which is operated under the ESO-ON agreement
} 
of the whole sample of pairs is similar to field galaxies. Are galaxies in dense environments more efficient in forming stars or do they have more fuel? How is the star formation efficiency correlated with the environment where galaxies reside? An essential step towards answering these open questions is to compare the properties of galaxies in dense environments and in the field.

\section{Sample Selection}

Surveys of the molecular gas content in galaxies have in general been done on samples which are far-infrared selected, or galaxies selected exclusively for belonging to clusters or groups, but with infrared selection criteria on top. In view of these biases plagueing existing samples we have selected our sample from the catalog by Maia et al. (1994) which contains objects in low and high density areas of the Southern sky. The selection of groups adopted by Maia et al. (1994) is similar to the methodology developed by Huchra \& Geller (1982) and by Maia et al (1989). The catalog was drawn from the SSRS (eg., da Costa et al. 1989). The groups are formed by the accumulation of galaxy pairs with a member in common. They have a density contrast $\delta \rho / \rho \geq 500$. This is equivalent to densities larger than 18 galaxies/ $\mathrm{Mpc}^{3}$. The control sample (CS) is made up of galaxies which are not members of any group and which are situated in regions with $\delta \rho / \rho \leq 0.01$, i.e. less than 0.0004 galaxies $/ \mathrm{Mpc}^{3}$. The high density sample (HDS) is formed by galaxies that are in groups of three or more members. We consider for the present analysis, only galaxies with velocities smaller than 5500 $\mathrm{kms}^{-1}$. Here we present the analysis of millimetric data of a subsample of 47 spiral galaxies, 22 in the HDS and 25 in the CS. Both samples are made of spiral galaxies later than $\mathrm{Sa}$ and earlier than $\mathrm{Sd}$.

\begin{tabular}{|c|c|c|c|c|c|}
\hline Sample ${ }^{f}$ & $\begin{array}{l}\mathrm{L}_{\mathrm{FIR}} / \mathrm{M}_{\mathrm{H}_{2}} \\
\mathrm{~L}_{\odot} / \mathrm{M}_{\odot}\end{array}$ & $\begin{array}{l}\mathrm{M}_{\mathrm{H}_{2}} / \mathrm{L}_{\mathrm{B}} \\
\mathrm{M}_{\odot} / \mathrm{L}_{\odot}\end{array}$ & $\begin{array}{l}\mathrm{L}_{\mathrm{FIR}} / \mathrm{L}_{\mathrm{B}} \\
\mathrm{L}_{\odot} / \mathrm{L}_{\odot}\end{array}$ & $\begin{array}{l}\mathrm{M}_{\mathrm{H}_{2}}+\mathrm{M}_{\mathrm{HI}} / \mathrm{L}_{\mathrm{B}} \\
\mathrm{M}_{\odot} / \mathrm{L}_{\odot}\end{array}$ & $\begin{array}{l}\mathrm{L}_{\mathrm{FIR}} /\left(\mathrm{M}_{\mathrm{H}_{2}}+\mathrm{M}_{\mathrm{H} 1}\right) \\
\mathrm{L}_{\odot} / \mathrm{M}_{\odot}\end{array}$ \\
\hline HDS & $0.74 \pm 0.31$ & $-1.09 \pm 0.39$ & $-0.35 \pm 0.32$ & $-0.35 \pm 0.29$ & $-0.01 \pm 0.42$ \\
\hline CS & $0.67 \pm 0.21$ & $-0.91 \pm 0.24$ & $-0.24 \pm 0.22$ & $-0.27 \pm 0.25$ & $0.00 \pm 0.26$ \\
\hline Pairs & $0.91 \pm 0.43$ & $-0.57 \pm 0.45$ & $0.33 \pm 0.48$ & $-0.47 \pm 0.31$ & $1.04 \pm 0.37$ \\
\hline CG & $0.39 \pm 0.33$ & $-0.61 \pm 0.39$ & $-0.16 \pm 0.45$ & $-0.42 \pm 0.22$ & $-0.02 \pm 0.40$ \\
\hline$C G_{\text {int.type }}$ & $0.37 \pm 0.40$ & $-0.66 \pm 0.35$ & $-0.29 \pm 0.49$ & $-0.14 \pm 0.31$ & $-0.12 \pm 0.40$ \\
\hline Starbursts & $1.24 \pm 0.39$ & $-0.61 \pm 0.43$ & $0.63 \pm 0.43$ & $-0.36 \pm 0.40$ & $0.91 \pm 0.39$ \\
\hline Clusters & $0.77 \pm 0.37$ & $-1.08 \pm 0.36$ & $-0.31 \pm 0.40$ & $-0.73 \pm 0.34$ & $0.42 \pm 0.35$ \\
\hline
\end{tabular}

Table 1. Logarithimic diagnostics are given above, where ${ }^{\dagger}$ HDS is our high density sample, CS is our control sample of isolated galaxies, Pairs, CGs, Starbursts and Clusters are from Leon et al. (1998). $\mathrm{CG}_{\text {int.type }}$ are $\mathrm{CG}$ galaxies later than $\mathrm{Sa}$ and earlier than $\mathrm{Sd}$ from Leon et al (1998).

\section{The Data}

Milimetric observations were carried out at the Swedish-ESO (SEST) $15 \mathrm{~m}$ radiotelescope at La Silla. We used the SESIS 100 receiver at $115 \mathrm{GHz}\left({ }^{12} \mathrm{CO}\right.$ (1-0)) and the IRAM 115 and IRAM 230 receivers at $115 \mathrm{GHz}\left({ }^{12} \mathrm{CO}(1-0)\right)$ and $230 \mathrm{GHz}\left({ }^{12} \mathrm{CO}(2-1)\right)$, respectively. All galaxies were observed at the central 

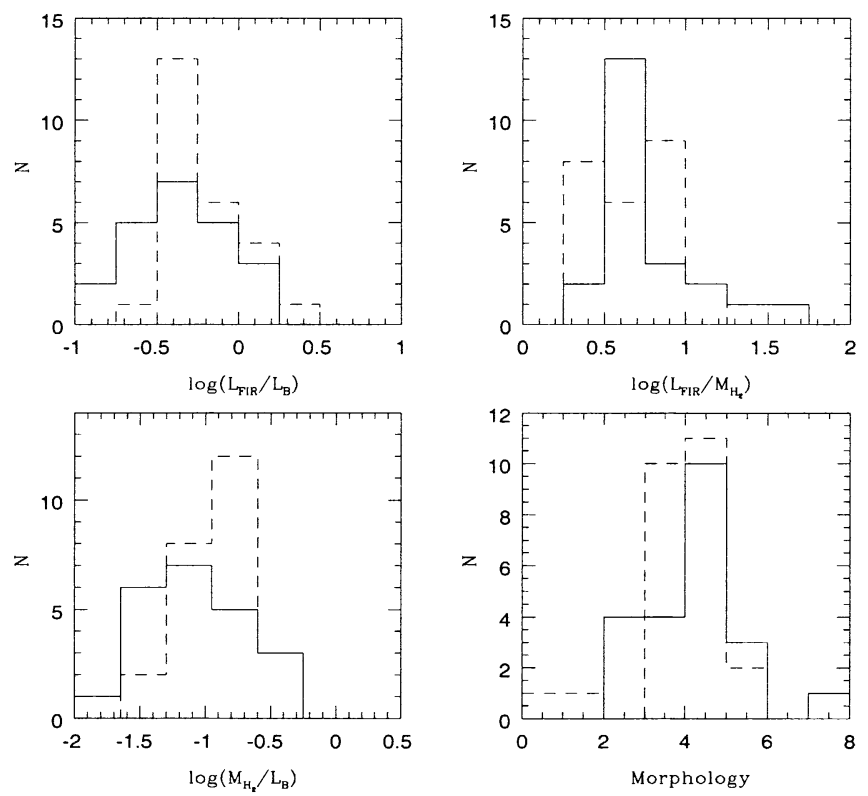

Figure 1. Distribution of physical parameters. Morphological types are: $1=\mathrm{Sa}, 2=\mathrm{Sa}-\mathrm{b}, 3=\mathrm{Sb}, 4=\mathrm{Sb}-\mathrm{c}, 5=\mathrm{S} . . ., 6=\mathrm{Sc}, \mathrm{Sc}-\mathrm{d}, 7=\mathrm{S} . . / \mathrm{Irr}$, $8=\mathrm{Sd}$. Full line is for the HDS and dashed line is for the CS.

(1-0)) and the IRAM 115 and IRAM 230 receivers at $115 \mathrm{GHz}\left({ }^{12} \mathrm{CO}(1-0)\right)$ and $230 \mathrm{GHz}\left({ }^{12} \mathrm{CO}(2-1)\right)$, respectively. All galaxies were observed at the central optical coordinate. Integration times were 2-3 hours depending on the signal-tonoise achieved. The spectra were reduced with the CLASS package (Forveille et al. 1990), and CO intensities were calculated by using the main-beam efficiency values of 0.7 and 0.5 for $115 \mathrm{GHz}$ and $230 \mathrm{GHz}$, respectively. Molecular gas masses were estimated from the line profile, using a $\mathrm{N}_{\mathrm{H}_{2}} / \mathrm{I}_{\mathrm{CO}}$ conversion ratio of $3 \times 10^{20} \mathrm{~cm}^{-2}\left(\mathrm{~K} \mathrm{kms}^{-1}\right)$. Besides the $\mathrm{CO}$ observations we made use of optical spectra obtained at ESO $1.52 \mathrm{~m}$ telescope, and HI and FIR from the literature.

\section{Analysis}

Several observable quantities are used as diagnostic of possible environmental effects such as the amount of molecular gas, dust temperature, star formation rate. In Fig. 1 is displayed the distribution of FIR luminosity, the amount of molecular gas and morphology for both samples.

The average values of the physical quantities are presented in table 1 which also contains comparisons for other samples such as of pairs, CGs, starbursts and cluster galaxies. The statistical significance of those distributions are examined by means of tests such as Kolmogorov-Smirnov (KS) and Student-t, displayed in table 2. 


\begin{tabular}{lcccc}
\hline \multicolumn{1}{c}{ Diagnostic } & $\mathrm{KS}$ & $\mathrm{KS}_{\mathrm{Pb}}$ & $\mathrm{T}$ & $\mathrm{T}_{\mathrm{Pb}}$ \\
\hline Morphology & 0.20 & 0.74 & -0.52 & 0.60 \\
$\log \left(\mathrm{M}_{\mathrm{H}_{2}} / \mathrm{L}_{\mathrm{B}}\right)$ & 0.33 & 0.16 & -1.89 & 0.07 \\
$\log \left(\mathrm{L}_{\mathrm{FIR}} / \mathrm{L}_{\mathrm{B}}\right)$ & 0.28 & 0.33 & -1.43 & 0.16 \\
$\log \left(\mathrm{L}_{\mathrm{FIR}} / \mathrm{M}_{\mathrm{H}_{2}}\right)$ & 0.23 & 0.57 & 0.85 & 0.40 \\
$\log \left(\mathrm{M}_{\mathrm{HI}} / \mathrm{L}_{\mathrm{B}}\right)$ & 0.31 & 0.42 & 0.45 & 0.65 \\
$\log \left(\mathrm{M}_{\mathrm{H}_{2}}+\mathrm{M}_{\mathrm{HI}} / \mathrm{L}_{\mathrm{B}}\right)$ & 0.42 & 0.09 & -1.19 & 0.24 \\
$\log \left(\mathrm{L}_{\mathrm{FIR}} / \mathrm{M}_{\mathrm{H}_{2}}+\mathrm{M}_{\mathrm{HI}}\right)$ & 0.27 & 0.55 & 0.16 & 0.87 \\
$\log \left(\mathrm{M}_{\mathrm{HI}} / \mathrm{M}_{\mathrm{H}_{2}}\right)$ & 0.40 & 0.12 & 0.87 & 0.39 \\
\hline
\end{tabular}

Table 2. Statistical Values. KS and T are Kolmogorov-Smirnov and Student tests. $\mathrm{KS}_{\mathrm{Pb}}$ and $\mathrm{T}_{\mathrm{Pb}}$ are their respective probabilities.

\section{Conclusions}

Based on the distributions and statistical tests of the pysical parameters evaluated, we can say that: (a) HDS spirals have overall less molecular gas per blue luminosity than the CS spirals; (b) the current star formation rate $\log \left(\mathrm{L}_{\mathrm{FIR}} / \mathrm{L}_{\mathrm{B}}\right)$ is lower, on average, in HDS than for CS objects; (c) the total gas content $\log \left(\mathrm{HI}+\mathrm{H}_{2}\right)$ is lower in the HDS galaxies; (d) there is more atomic gas, or higher atomic gas fraction $\log \left(\mathrm{M}_{\mathrm{HI}} / \mathrm{M}_{\mathrm{H}_{2}}\right)$ in the HDS sample and (e) HDS galaxies have similar SFE values as the ones in the field.

\section{References}

Boselli, A., Mendes de Oliveira, C., Balkowski, C., Cayatte, V., \& Casoli, F. 1996, A\&A 314, 738

Casoli, F., Boisse, P., Combes, F. \& Dupraz, C. 1991, A\&A 249, 359

Casoli, F., Dickey, J., Kazes, I., Boselli, A., Gavazzi, P., \& Baumgardt, K. 1996, A\&A 309, 43

Combes, F., Prugniel, P., Rampazzo, R., \& Sulentic, J.W. 1994, A\&A 281, 725 da Costa, L.N., Pellegrini, P.S., Willmer, C., de Carvalho, R., Maia, M., Latham, D.W., \& Geary, J.C. 1989, AJ 97, 315

Forveille, T., Guilloteau, S., \& Lucas, R. 1990, IRAM internal report

Horellou, C., Casoli, F., Combes, F., \& Dupraz, C. 1995, A\&A 298, 743

Huchra, J. P., \& Geller, M.J. 1982, ApJ 257, 423

Leon, S., Combes, F., \& Menon, T.K. 1998, AA, 330, 37

Maia, M.A.G., da Costa, L.N., \& Latham, D.W. 1989, ApJS 69, 809

Maia, M.A.G., Pastoriza, M.G., Bica, E., Dottori, H. 1994, ApJS 93, 425

Mendes de Oliveira, C., \& Hickson, P. 1994, ApJ 427, 684

Sulentic, J.W., \& de Mello Rabaça, D.F. 1993, ApJ 410, 520

Williams, B.A., \& Rood, H.J. 1987, ApJS 63, 265 